\title{
Synthesis of Jet Fuel from Glycerol and tert-Butyl Alcohol under Microwave Irradiation
}

\author{
Ruru Zhou ${ }^{a, \dagger}$, Yuanyuan Jiang ${ }^{a, \dagger}$, Boyong Ye ${ }^{a}$, Huaiyuan Zhao ${ }^{a}$, Zhaoyin Hou ${ }^{a, b, *}$ \\ ${ }^{a}$ Key Laboratory of Biomass Chemical Engineering of Ministry of Education, Department of \\ Chemistry, Zhejiang University, Hangzhou 310028, China. \\ ${ }^{b}$ Center of Chemistry for Frontier Technologies, Department of Chemistry, Zhejiang University, \\ Hangzhou 310028, China.
}

\section{Etherification of glycerol with TBA with different energy supply methods}

Conventional heating experiments were conducted in a flask which was heated in an oil bath equipped with magnetic stirring (500 rpm) and a condenser. In a typical run, $10.86 \mathrm{mmol}$ glycerol, $88.68 \mathrm{mmol}$ TBA and $0.05 \mathrm{~g}$ catalyst were placed in a round bottom flask.

Ultrasound wave experiments were performed using a KQ-300DB ultrasonic instrument (Kunshan Ultrasonic Instrument Co. Lt, China) with ultrasound wave frequency of $40 \mathrm{KHz}$ produced by a $300 \mathrm{~W}$ generator through a transducer. In a typical run, $10.86 \mathrm{mmol}$ glycerol, $86.88 \mathrm{mmol}$ TBA and $0.05 \mathrm{~g}$ catalyst were placed in a round bottom flask.

Ultraviolet irradiation experiments were carried out under the irradiation of ultraviolet with a wave length of $365 \mathrm{~nm}$ and a power of $200 \mathrm{~W}$. In a typical run, $10.86 \mathrm{mmol}$ glycerol, 86.88 mmol TBA and $0.05 \mathrm{~g}$ catalyst were placed in a round bottom flask. The flask was placed in an 
water bath with magnetic stirring $(500 \mathrm{rpm})$ to reach required reaction temperature.

Ball milling experiments were implemented using a planetary ball mill (Retsch PM100,

Germany). In a typical run, $10.86 \mathrm{mmol}$ glycerol, $86.88 \mathrm{mmol}$ TBA and $0.05 \mathrm{~g}$ catalyst were placed in a $50 \mathrm{~mL}$ agate jar, which also contained $25 \mathrm{~g}$ agate balls ( $5 \mathrm{~mm}$ in diameter).

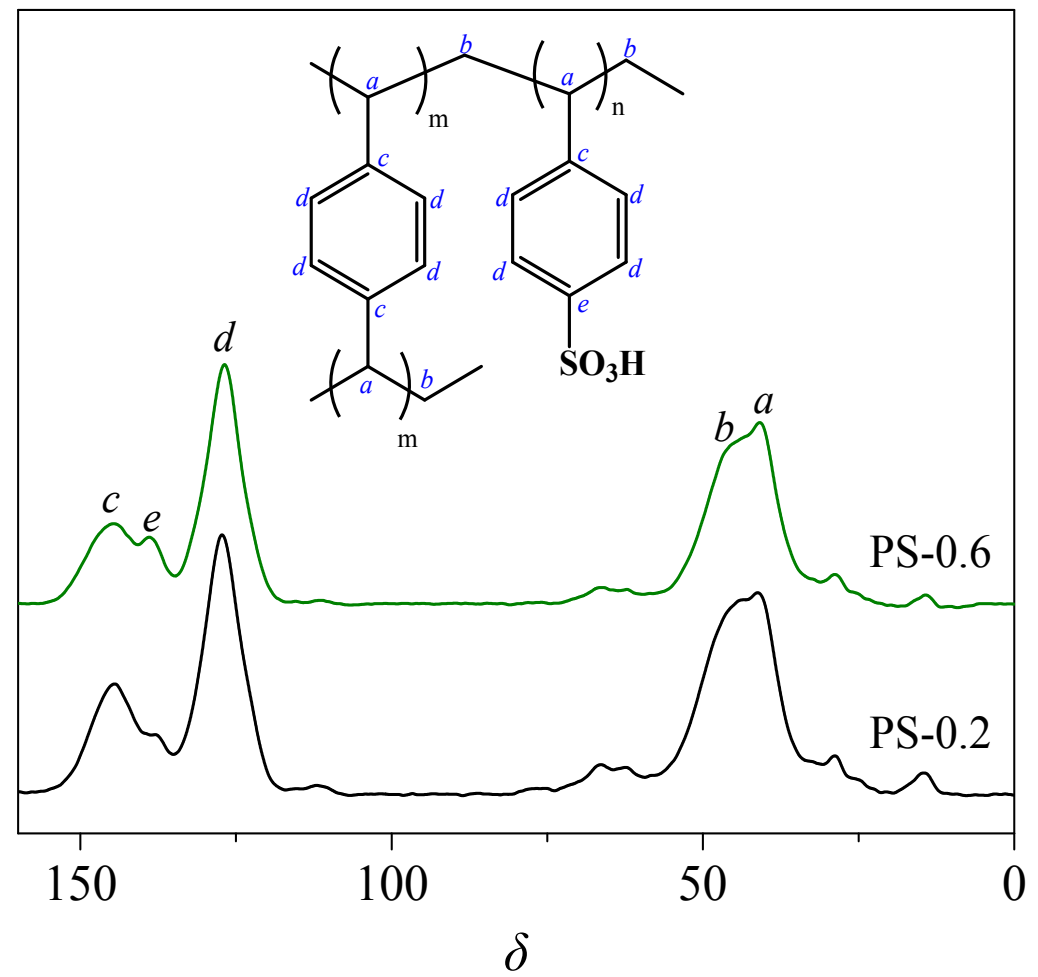

Figure S1. ${ }^{13} \mathrm{C}$ solid-state NMR spectra of PS-0.2 and PS-0.6. 

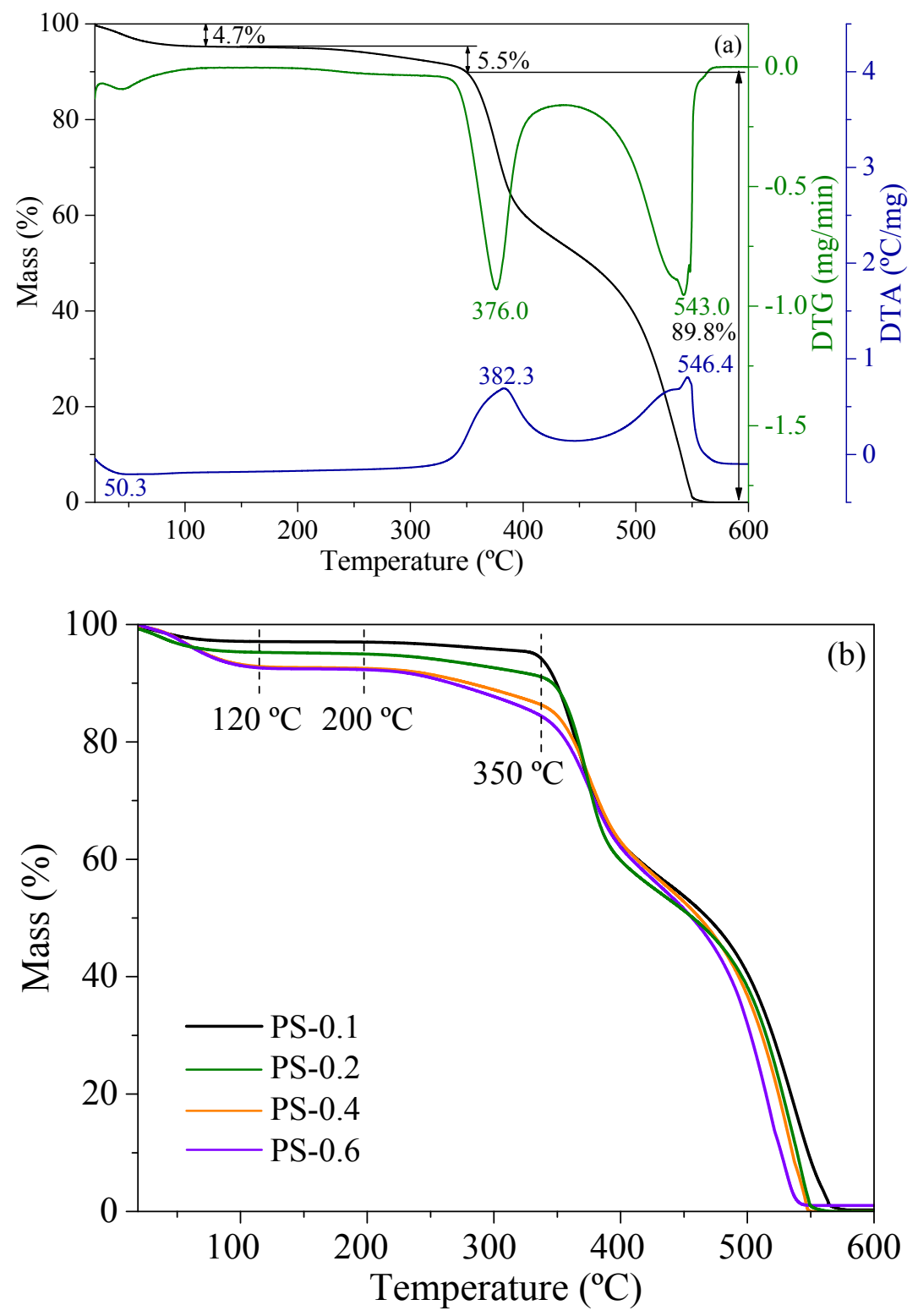

Figure S2. TG-DTG-DTA curves of PS-0.2 (a) and TG curves of PS- $x$ (b). 

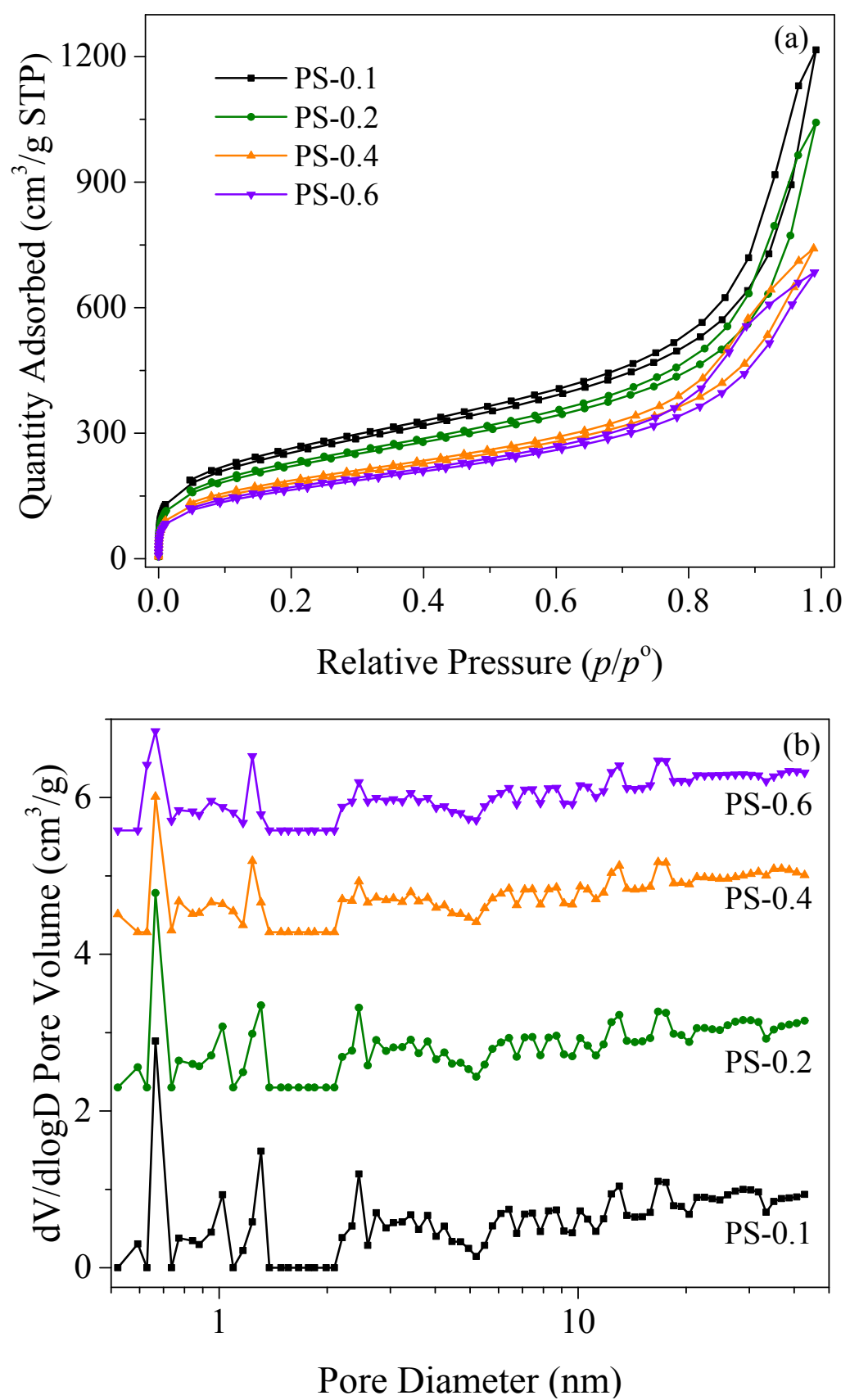

Figure S3. $\mathrm{N}_{2}$ adsorption-desorption isotherms (a) and pore size distribution curves (b) of PS- $x$. 

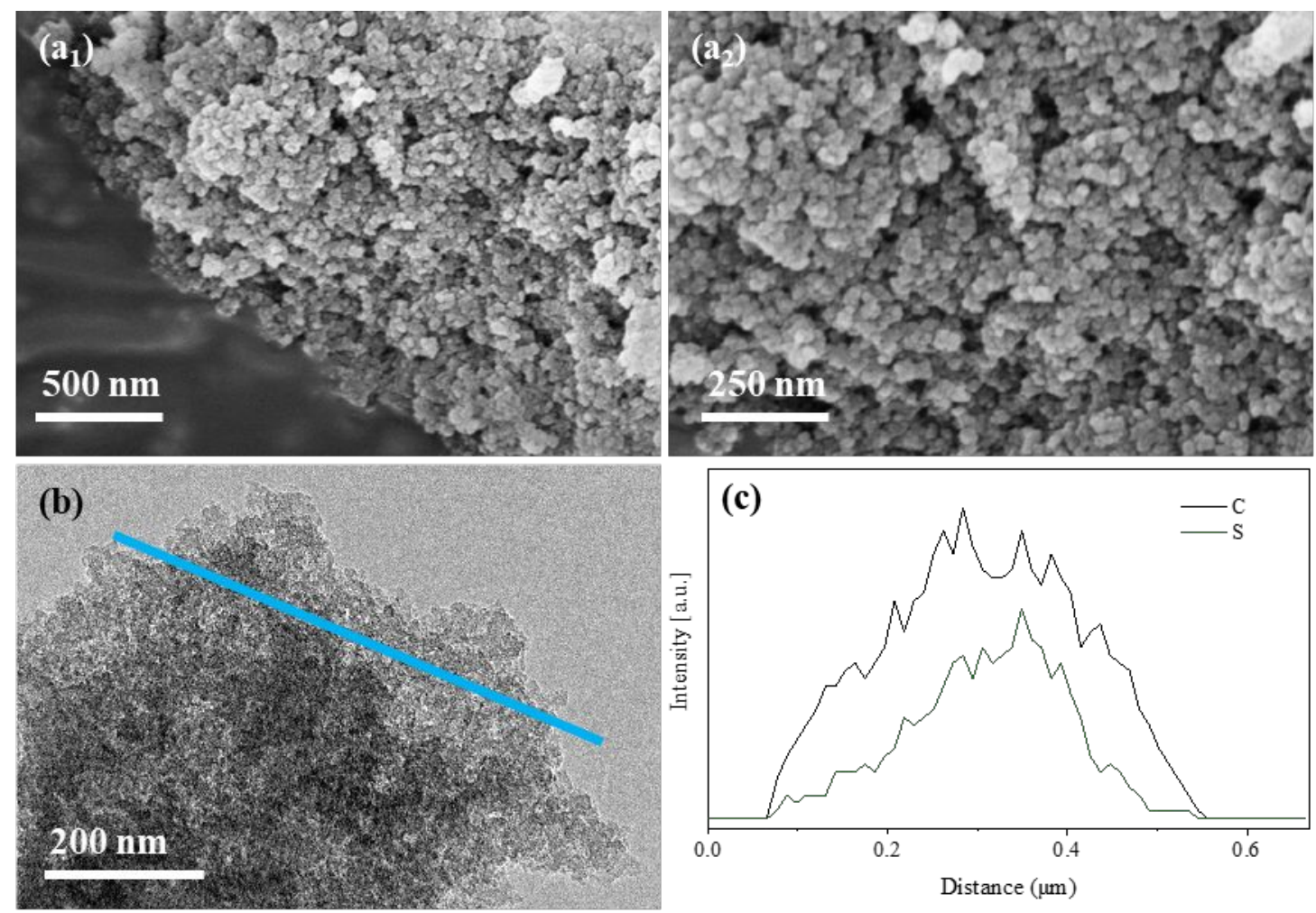

Figure S4. SEM $\left(a_{1}, a_{2}\right)$, TEM images (b) and line-EDS (c) of PS-0.2. 

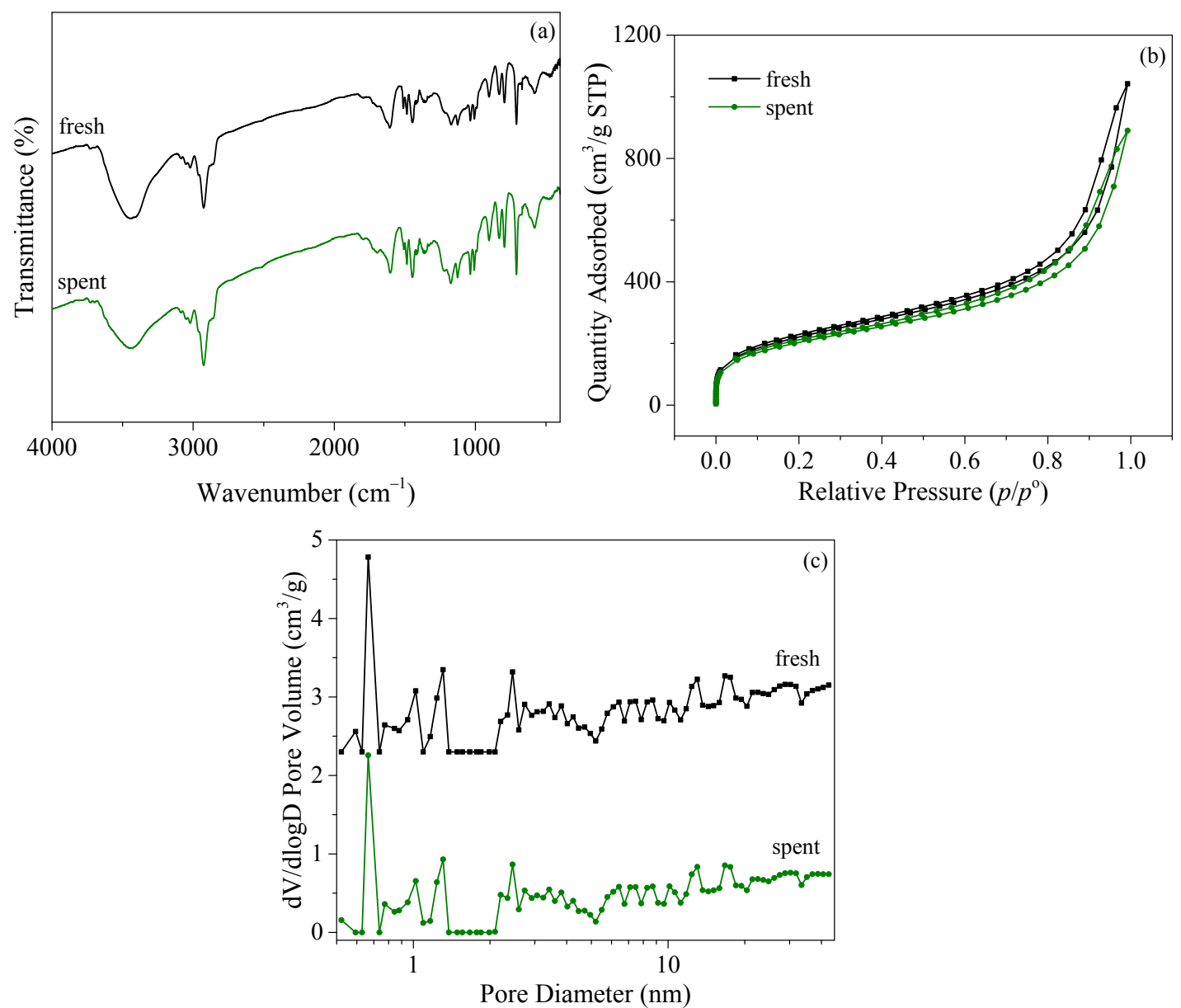

Figure S5. FTIR spectra (a), $\mathrm{N}_{2}$ adsorption-desorption isotherms (b) and pore size distribution curves (c) of fresh and spent PS-0.2. 


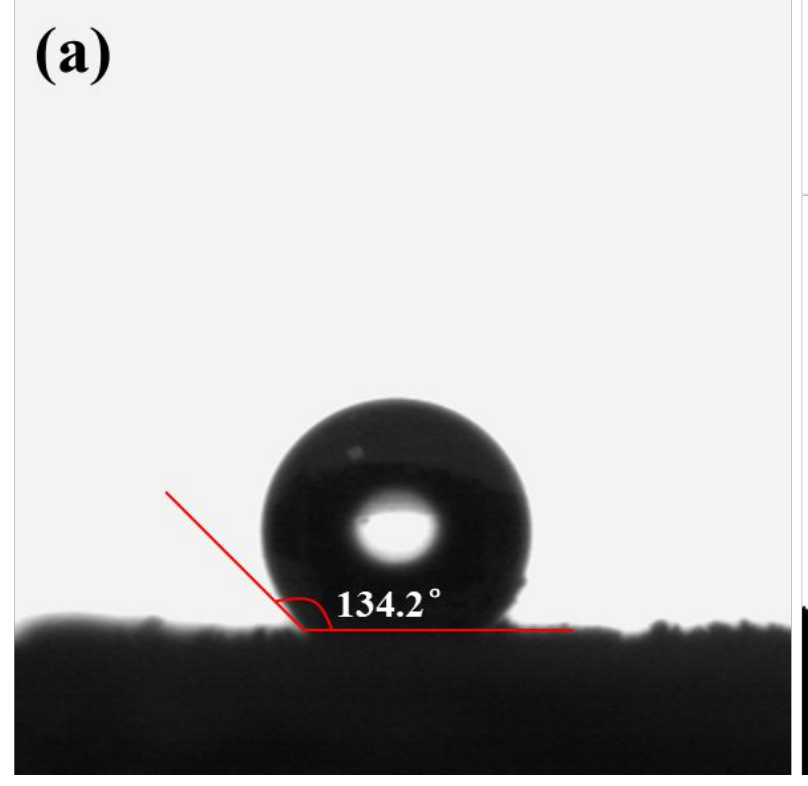

(b)

Figure S6. Water contact angle of fresh (a) and spent (b) PS-0.2.

Table S1. Surface element composition of fresh and recycled PS-0.2.

\begin{tabular}{lccc}
\hline \multirow{2}{*}{ Sample } & \multicolumn{3}{c}{ Surface composition $(\%)^{a}$} \\
\cline { 2 - 4 } & $\mathrm{C}$ & $\mathrm{O}$ & $\mathrm{S}$ \\
\hline Fresh sample & 89.17 & 10.48 & 0.35 \\
Spent sample & 89.29 & 10.40 & 0.31 \\
\hline$a$ Detected by XPS & &
\end{tabular}




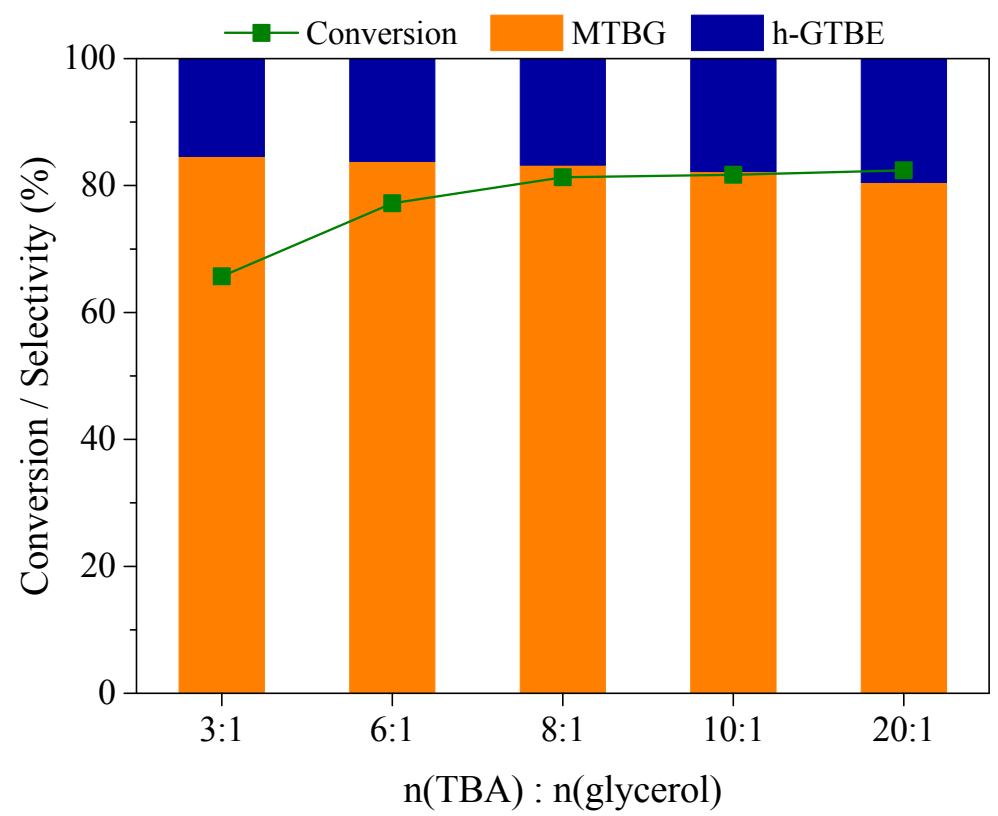

Figure S7. Etherification of glycerol and TBA at different ratio of TBA/glycerol in feed. Reaction conditions: $10.86 \mathrm{mmol}$ glycerol, $0.05 \mathrm{~g}$ PS- $0.2,80^{\circ} \mathrm{C}, 2450 \mathrm{MHz}, 300 \mathrm{~W}, 1.5 \mathrm{~h}$.

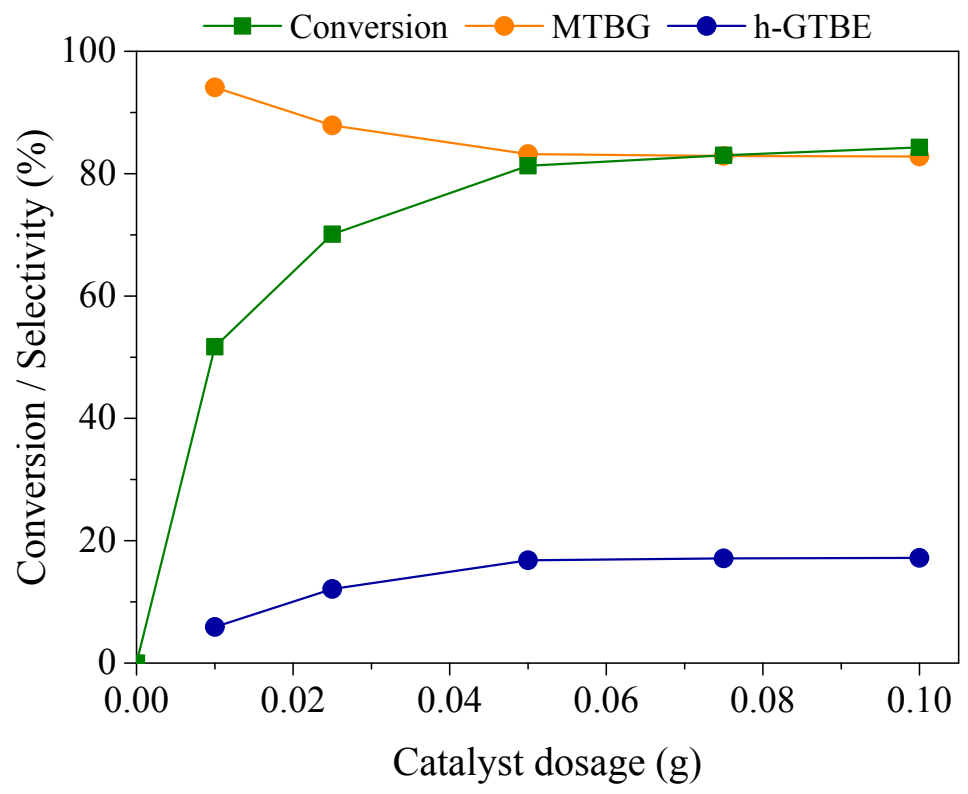

Figure S8. Etherification of glycerol and TBA over different catalyst dosage of PS-0.2. Reaction conditions: $10.86 \mathrm{mmol}$ glycerol, $86.88 \mathrm{mmol}$ TBA, $80{ }^{\circ} \mathrm{C}, 2450 \mathrm{MHz}, 300 \mathrm{~W}, 1.5 \mathrm{~h}$. 


\section{Reaction kinetics}

(1) Adsorption of glycerol is the rate determining step:

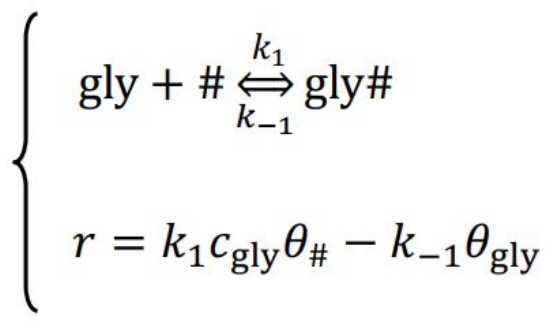

As $\theta_{\#}>>\theta_{\text {gly }}$ and $\theta_{\#}+\theta_{\text {gly }}=1$, it could be assumed that $\theta_{\#} \approx 1$.

The overall reaction rate could be expressed in

$$
r \approx k c_{\text {gly }}
$$

where $r$ refers to the consumption rate of glycerol.

(2) Surface reaction between adsorbed glycerol and TBA is the rate determining step:

$$
r=-\frac{\mathrm{d} c_{\mathrm{gly}}}{\mathrm{d} t}=k \theta_{\mathrm{gly}} \theta_{\mathrm{TBA}}=\frac{k K_{\mathrm{gly}} c_{\mathrm{gly}} K_{\mathrm{TBA}} c_{\mathrm{TBA}}}{\left(1+K_{\mathrm{gly}} c_{\mathrm{gly}}+K_{\mathrm{TBA}} c_{\mathrm{TBA}}\right)^{2}}
$$

where $r$ refers to the consumption rate of glycerol.

As the amount of TBA in feed was excessively stoichiometric in the reaction mixture $(\mathrm{n}(\mathrm{TBA}): \mathrm{n}($ glycerol $)=8: 1)$, that is $c_{\text {gly }}<<c_{\mathrm{TBA}}$. This equation could be simplified:

$$
r \approx k K_{\mathrm{gly}} c_{\mathrm{gly}} \frac{K_{\mathrm{TBA}} c_{\mathrm{TBA}}}{1+K_{\mathrm{TBA}} c_{\mathrm{TBA}}} \approx k^{\prime} c_{\mathrm{gly}}
$$

Above results indicated that the consumption rate of glycerol over PS-0.2 could be proportional to the concentration of glycerol as follows. 


$$
r \approx k c_{\mathrm{gly}}
$$

\section{Reaction kinetics over PS-0.2:}
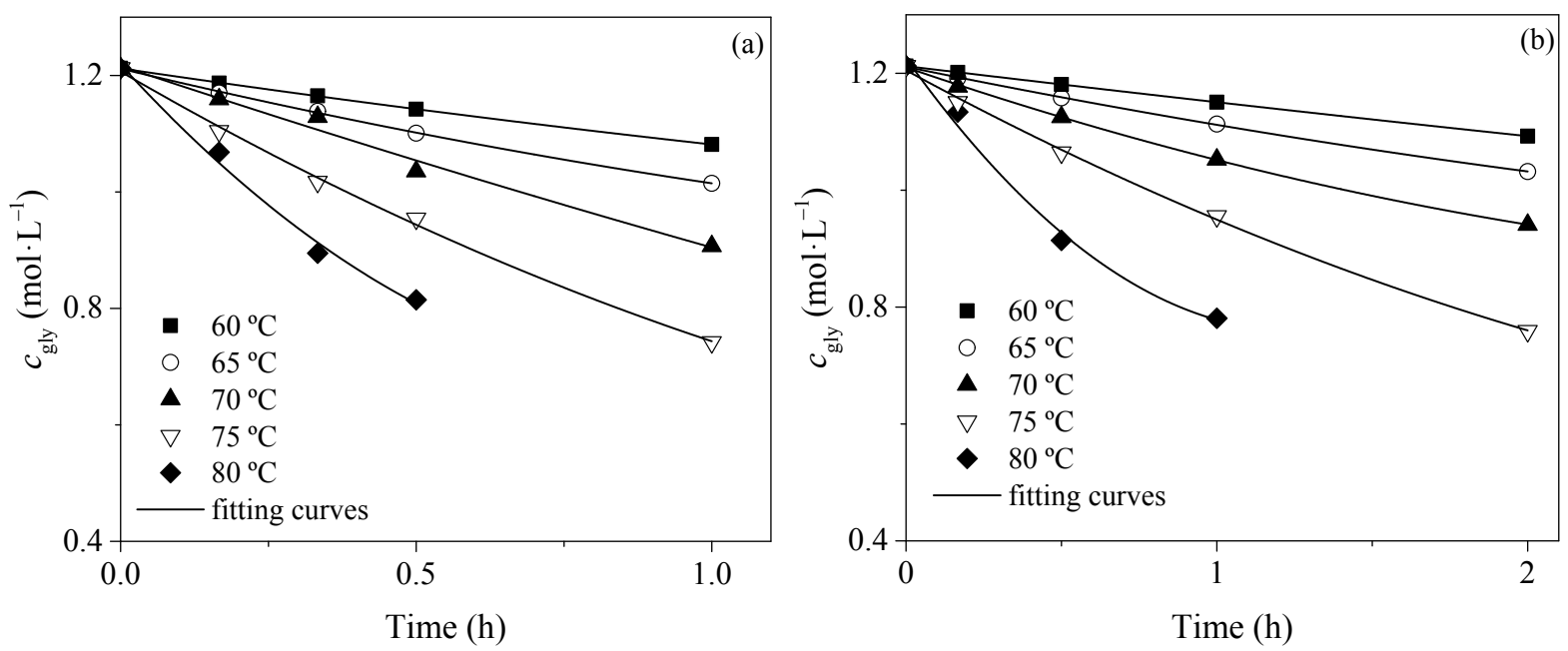

Figure S9. Kinetic experimental data under microwave irradiation (a) and conventional heating

(b) over PS-0.2.

Reaction conditions: $10.86 \mathrm{mmol}$ glycerol, $86.88 \mathrm{mmol}$ TBA, 0.05 g PS-0.2 (Microwave irradiation: 2450 MHz, $300 \mathrm{~W}$; Conventional heating: $500 \mathrm{rpm})$.
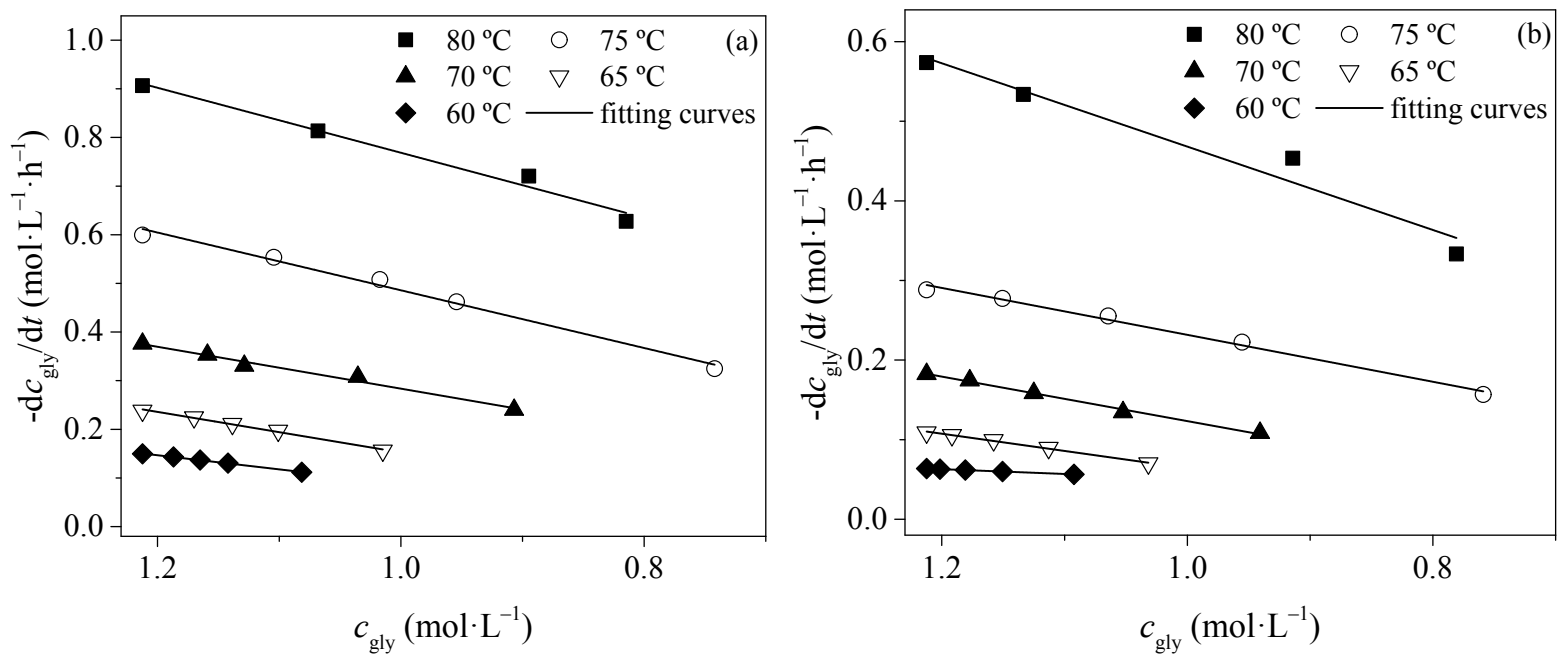
Figure S10. Linear fitting of $\left(-\mathrm{d} c_{\text {gly }} / \mathrm{d} t\right)-c_{\text {gly }}$ curves under microwave irradiation (a) and conventional heating (b) over PS-0.2.

Reaction conditions: $10.86 \mathrm{mmol}$ glycerol, $86.88 \mathrm{mmol}$ TBA, $0.05 \mathrm{~g}$ PS-0.2 (Microwave irradiation: 2450 $\mathrm{MHz}, 300 \mathrm{~W}$; Conventional heating: $500 \mathrm{rpm}$ ).
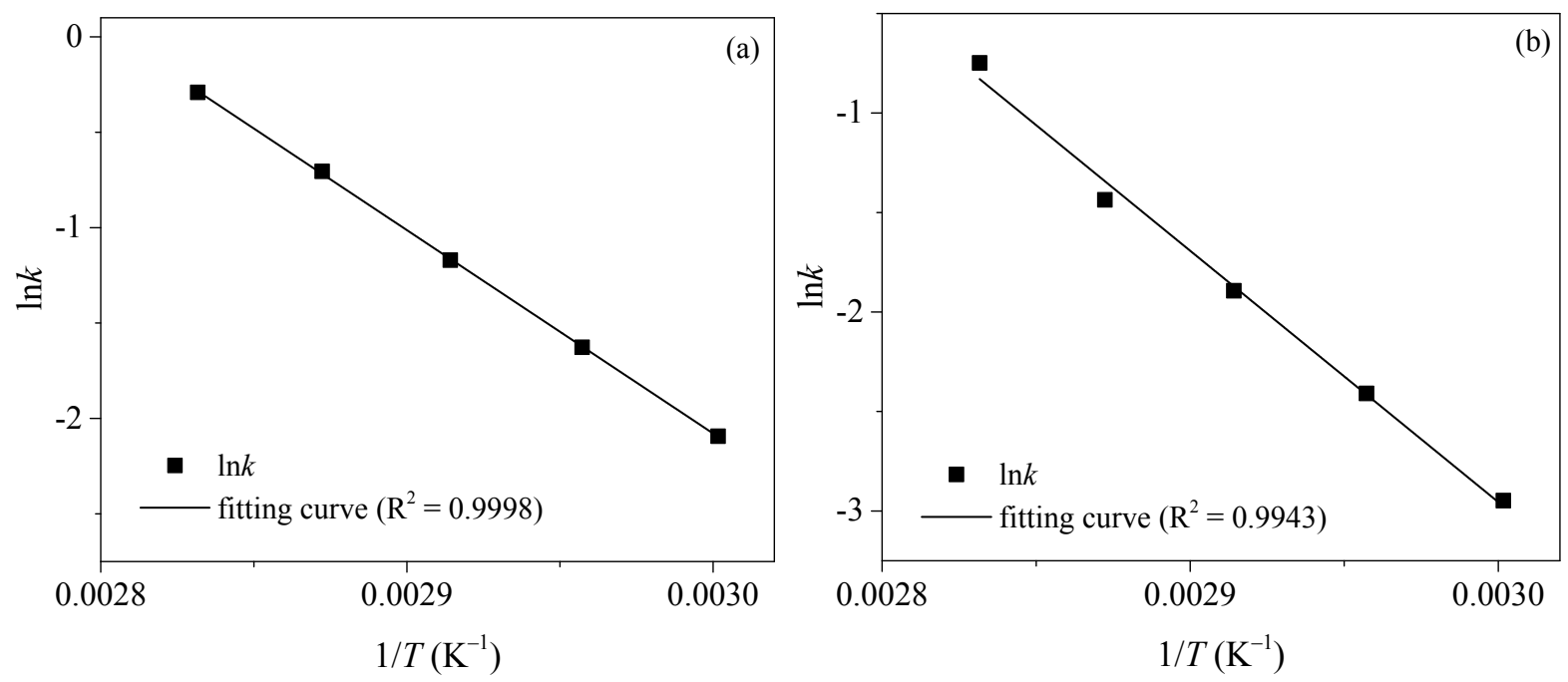

Figure S11. Linear fitting of $\ln k-1 / T$ curve under microwave irradiation (a) and conventional heating (b) over PS-0.2.

Reaction conditions: $10.86 \mathrm{mmol}$ glycerol, $86.88 \mathrm{mmol}$ TBA, 0.05 g PS-0.2 (Microwave irradiation: 2450 MHz, $300 \mathrm{~W}$; Conventional heating: $500 \mathrm{rpm}$ ). 


\section{Reaction kinetics over Amberlyst ${ }^{\circledR}-45$ :}
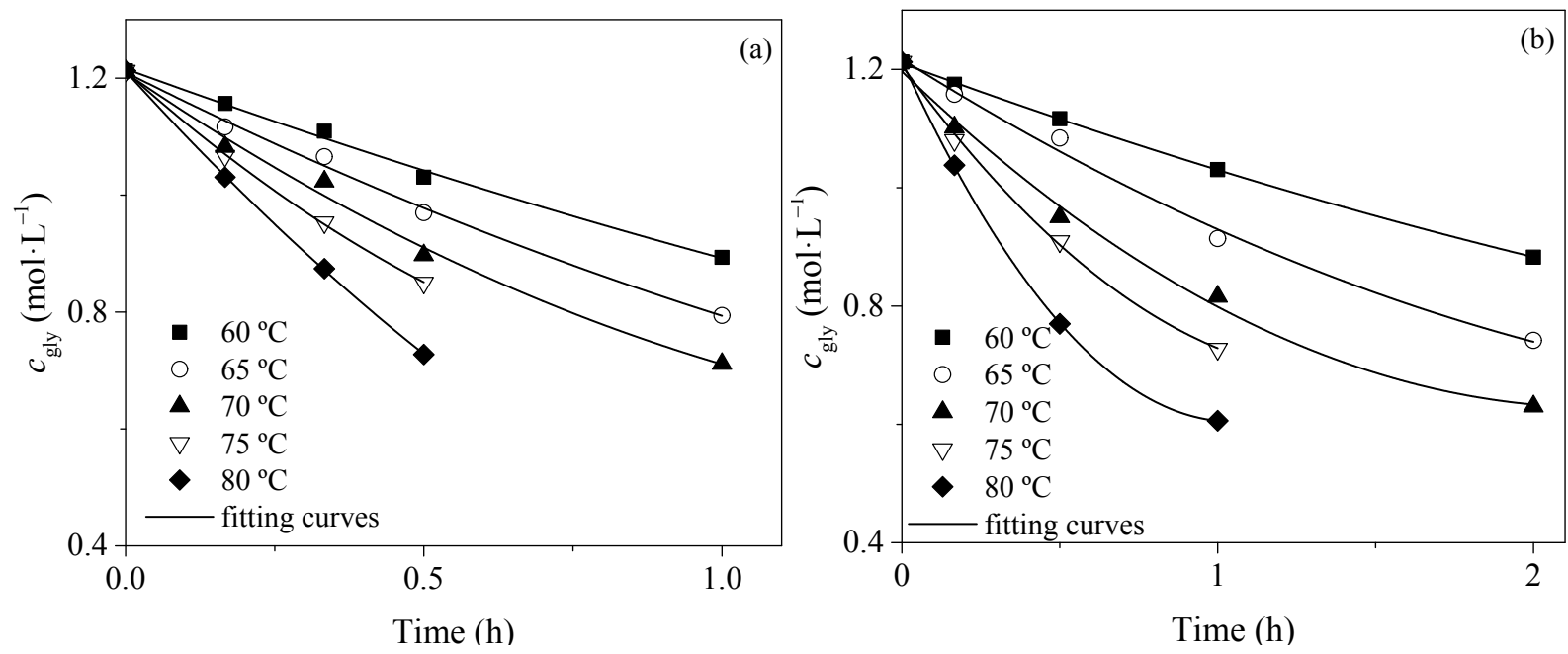

Figure S12. Kinetic experimental data under microwave irradiation (a) and conventional heating

(b) over Amberlyst ${ }^{\circledR}-45$.

Reaction conditions: $10.86 \mathrm{mmol}$ glycerol, $86.88 \mathrm{mmol}$ TBA, $0.045 \mathrm{mmol}$ acid sites (Microwave irradiation: $2450 \mathrm{MHz}, 300 \mathrm{~W}$; Conventional heating: $500 \mathrm{rpm}$ ).
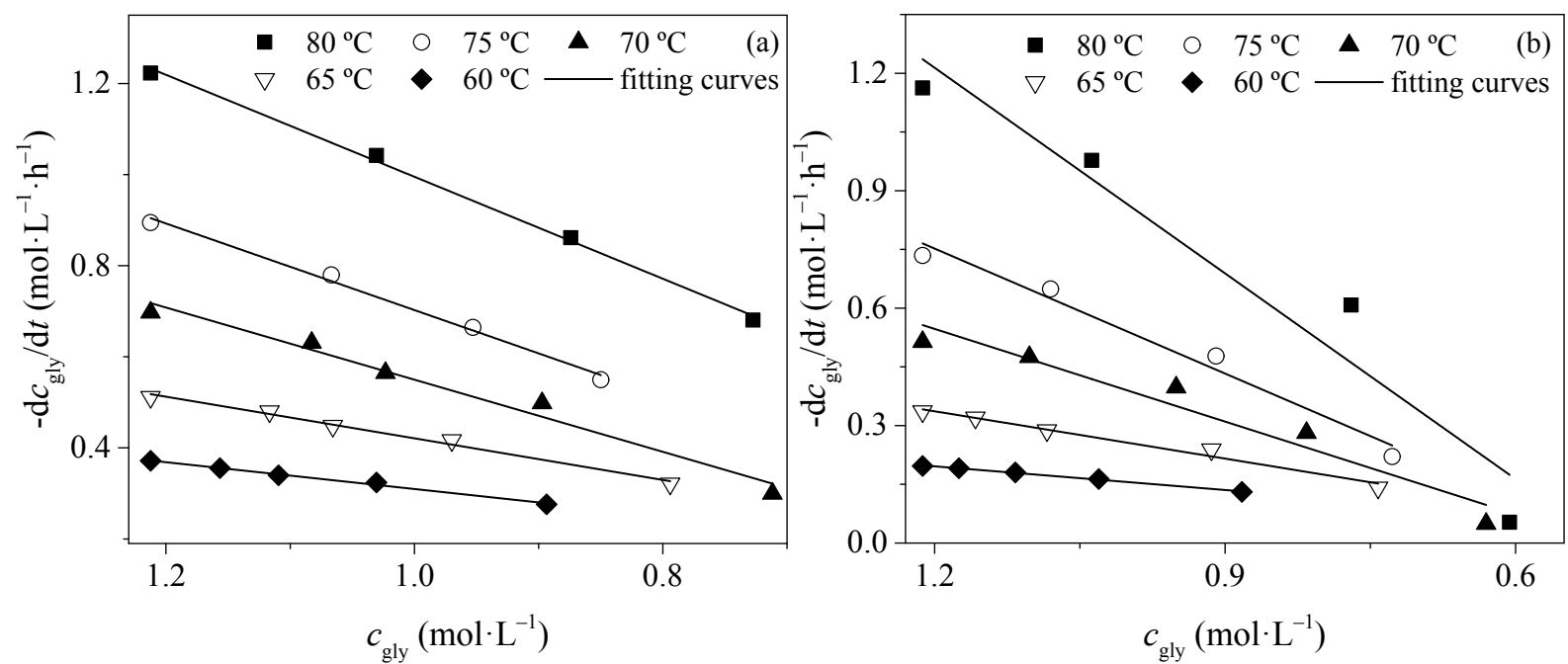

Figure S13. Linear fitting of $\left(-\mathrm{d} c_{\text {gly }} / \mathrm{d} t\right)-c_{\text {gly }}$ curves under microwave irradiation (a) and conventional heating (b) over Amberlyst ${ }^{\circledR}$-45. 
Reaction conditions: $10.86 \mathrm{mmol}$ glycerol, $86.88 \mathrm{mmol}$ TBA, $0.045 \mathrm{mmol}$ acid sites (Microwave irradiation: $2450 \mathrm{MHz}, 300 \mathrm{~W}$; Conventional heating: $500 \mathrm{rpm}$ ).
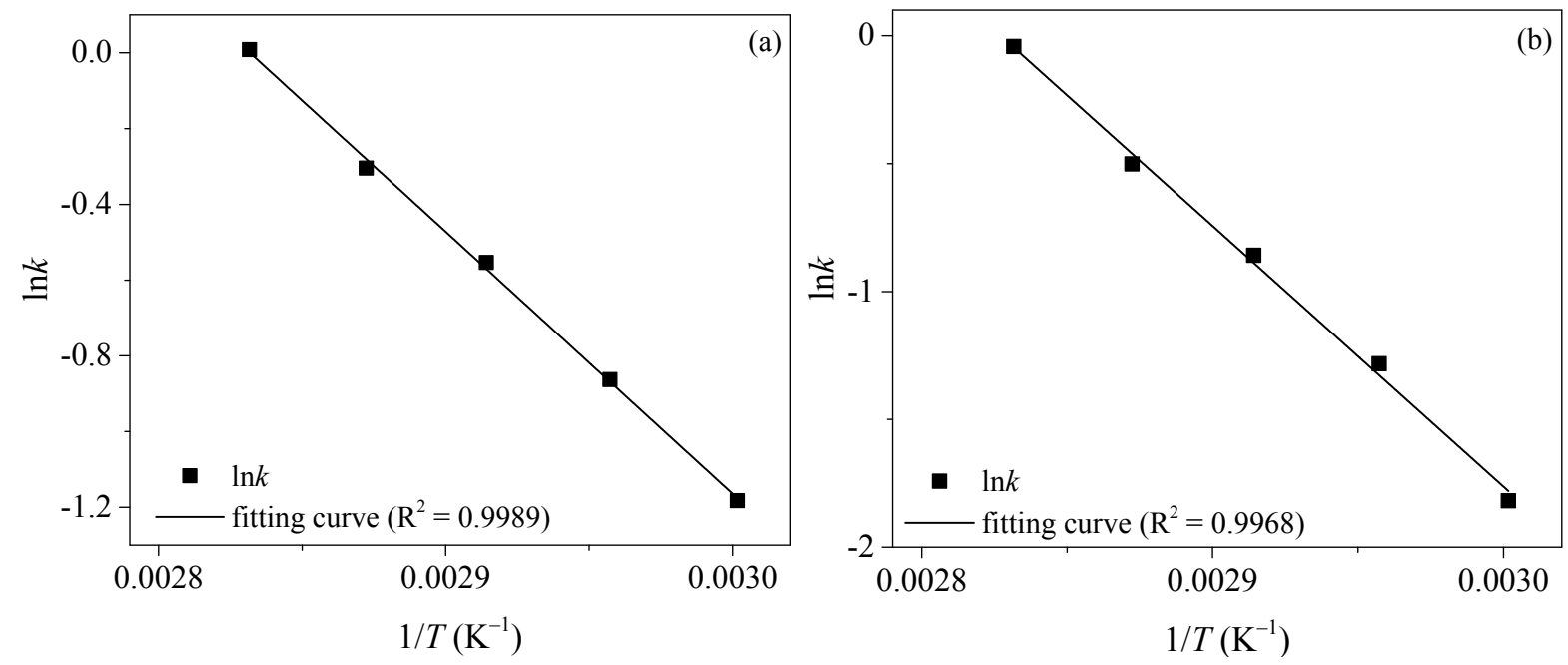

Figure S14. Linear fitting of $\ln k-1 / T$ curve under microwave irradiation (a) and conventional heating (b) over Amberlyst ${ }^{\circledR}$-45.

Reaction conditions: $10.86 \mathrm{mmol}$ glycerol, $86.88 \mathrm{mmol}$ TBA, $0.045 \mathrm{mmol}$ acid sites (Microwave irradiation: $2450 \mathrm{MHz}, 300 \mathrm{~W}$; Conventional heating: $500 \mathrm{rpm}$ ).

Table S2. Kinetic parameters of etherification of glycerol and TBA over Amberlyst ${ }^{\circledR}-45$.

\begin{tabular}{ccccc}
\hline \multirow{2}{*}{$\begin{array}{c}\text { Temperature } \\
\left({ }^{\circ} \mathrm{C}\right)\end{array}$} & $k\left(\mathrm{~h}^{-1}\right)$ & Arrhenius equation & \multicolumn{2}{c}{ Conventional heating } \\
\cline { 2 - 5 } 80 & 1.009 & $\ln k=-6923.1 / T+19.61$ & 0.959 & $\ln k=-10205 / T+28.85$ \\
75 & 0.738 & & 0.606 & Arrhenius equation $^{a}$ \\
70 & 0.575 & $E_{a}=57.6 \mathrm{~kJ} / \mathrm{mol}$ & 0.424 & $E_{a}=84.8 \mathrm{~kJ} / \mathrm{mol}$ \\
65 & 0.422 & $A=3.268 \times 10^{8} \mathrm{~h}^{-1}$ & 0.277 & $A=3.387 \times 10^{12} \mathrm{~h}^{-1}$ \\
60 & 0.306 & & 0.162 & \\
\hline
\end{tabular}

${ }^{a}$ Arrhenius equation: $\ln k=-E_{a} / R T+\ln A$. 